\title{
A Study on Recent Advancements in MIMO-FBMC Systems
}

\author{
Bidyalaxmi Devi \\ Tensubam \\ Post Graduate Scholar \\ DIT University \\ ECE Department
}

\author{
Nongmaithem Lalleima \\ Chanu \\ Post Graduate Scholar \\ DIT University \\ ECE Department
}

\author{
Sonika Singh, Ph.D \\ Associate Professor \\ DIT University \\ ECE Department
}

\begin{abstract}
Multicarrier (MC) techniques when combined with Multiple Input Multiple Output (MIMO) technology increases the throughput and QOS of the system. OFDM with its unique capability of converting the frequency selective channel into a set of frequency flat channel have proved to be one of the best multicarrier technique that can be applied to MIMO channels. However, OFDM suffers from Bandwidth loss due to the addition of cyclic prefix. Filter bank multicarrier (FBMC) modulation system on the other hand, is highly bandwidth efficient but its application to MIMO channels is still a subject of study to various researchers for the past decade. So far, only FMT based FBMC system offers same flexibility as OFDM in adopting MIMO techniques. This paper presents the various advancements or methods proposed to enhance the flexibility of FBMC systems (FMT,CMT,OFDM-OQAM) while adopting MIMO techniques mainly highlighting the ISI and ICI mitigation methods through improved decoding techniques, equalization schemes and modified FBMC schemes.
\end{abstract}

\section{Keywords}

MC, MIMO, FBMC, OFDM, OFDM-OQAM, QAM, FMT, CMT.

\section{INTRODUCTION}

Multicarreir modulation(MCM) techniques are the potential candidates taken into consideration to meet the growing demands of high data rate users. This demand can be more efficiently dealt with the combination of multicarrier techniques and MIMO technology, which uses multiple antennas at transmission and reception to boost the system performance without the requirement of additional bandwidth. This combination provides both efficiency and Quality of service(QOS) to the system.

One of the best MC techniques that can be jointly used with MIMO technology is OFDM. In OFDM, the fading at the subcarrier level is modelled flat which which facilitates the implementation of MIMO concept. However OFDM suffers from some serious drawbacks such as poor stopband attenuation, which necessitates tight synchronization and the need of a cyclic prefix to deal with the channel impulse response which accounts to the reduced bandwidth efficiency[1].

FBMC, an alternative MC technique with a better spectral localization overcomes the drawbacks of OFDM and offers several advantages such as increased spectral efficiency through the removal of cyclic prefix, lower spectral leakage, reduced sensitivity to doppler effects and also much relaxed synchronization requirements[2].
However, it turns out that, while the application of OFDM to MIMO channels is a straightforward task, direct application of FBMC to MIMO channels is a nontrivial task. Recently various studies have been done on the various FBMC systems namely Filtered Multitone (FMT), Cosine Modulated Multitone (CMT), Staggered Modulated Multitone (SMT) or OFDM-OQAM and many improvements have been proposed for all the systems with respect to their applications to MIMO channels. In FMT, the subcarriers do not overlap as it follows the conventional method of Frequency Division Multiplexing (FDM) and its application to MIMO channels is straightforward and can offer the same flexibility as OFDM in adopting MIMO techniques as the channel can be approximated by a flat gain over each subcarrier band. However, FMT suffers from bandwidth loss due to the guard bands employed. Whereas in CMT and SMT, the subcarrier overlap and offset QAM (OQAM) modulation is used, which preserves bandwidth. OQAM has the potential to outperform QAM modulation, however its main drawback is the inter symbol interference (ISI) and inter carrier interference (ICI) terms associated with the received data symbol received at the receiver. This paper presents the study of various methods introduced by various researchers which contributes in the extension of MIMO architectures to FBMC.

\section{MIMO FBMC-OQAM MODEL}

A MIMO FBMC-OQAM system with $N_{T}$ Transmit antennas and $N_{R}$ Receive antennas is considered [3] and the corresponding block diagram is shown in Fig.1. At the Msubchannel synthesis bank of the FBMC-OQAM transmitter, the discrete time baseband signal that corresponds to the $i^{\text {th }}$ transmit antenna can be expressed as-

$$
S_{m}^{i}=\sum_{K \in M_{L}} \sum_{n=-\infty}^{\infty} v_{K_{2} n}^{i} g_{K_{2} m-m \frac{M}{2}}
$$

Here, $v_{K n}^{\mathrm{i}}=d_{K n}^{\mathrm{i}} \theta_{K n}$ is the output of the OQAM modulator for the $K^{\text {th }}$ subchannel at the $i^{\text {th }}$ transmit antenna and $M_{u}$ denotes the set of active subchannels. The transmitted symbols are assumed to be uncorrelated in space, time and frequency. The signals $S_{\mathrm{m}}^{\mathrm{i}}$, for $\mathrm{i}=1,2, \ldots \ldots, N_{\mathrm{t}}$, are then transmitted through a frequency selective MIMO channel which is assumed to be time invariant over the duration of a transmitted FBMC-OQAM frame. The multipath impulse response of the $p^{\text {th }}$ tap is given by 


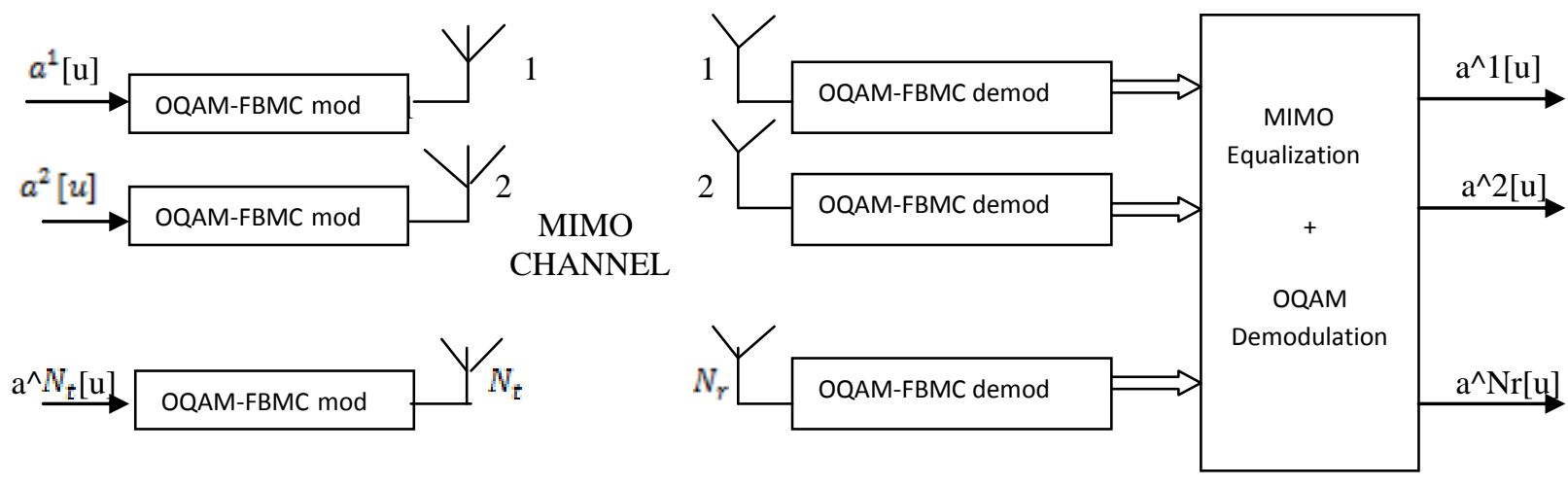

Fig 1: MIMO FBMC-OQAM system model [3]

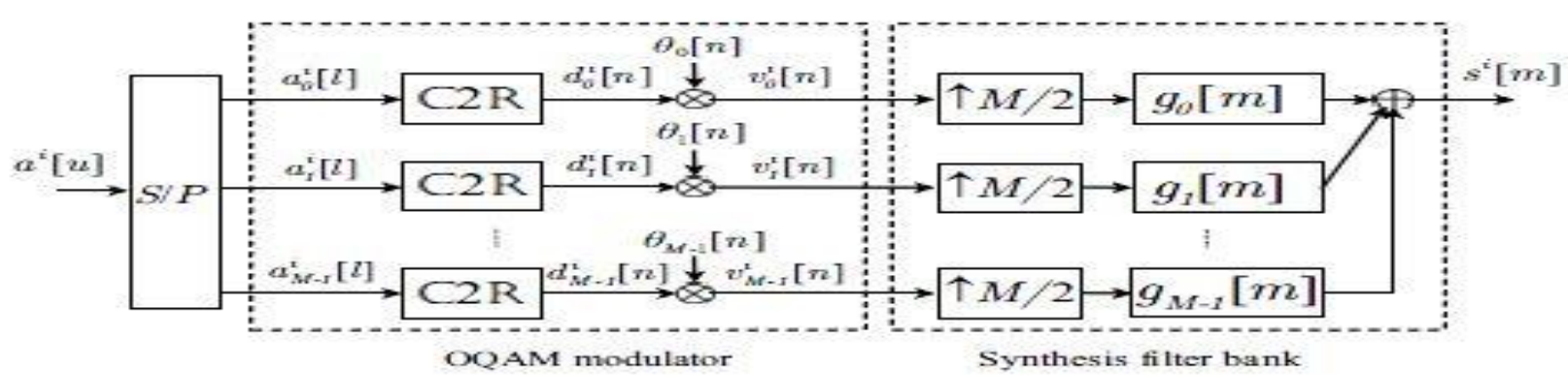

Fig 2: OQAM modulation and synthesis filter bank[3]
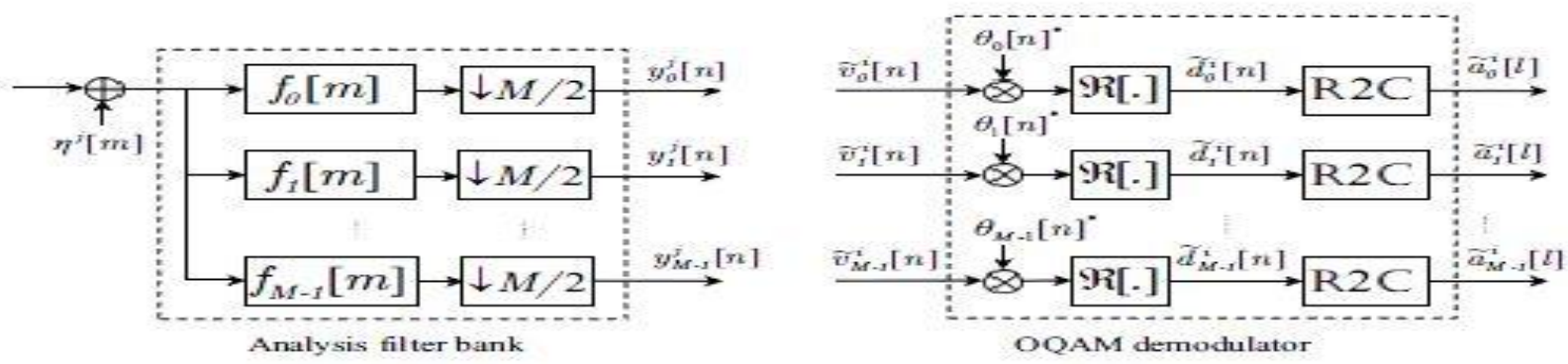

Fig 3: OQAM demodulation and analysis filter bank[3]

Table 1: Graphical representation of time-frequency response of FBMC system from the design of Bellanger in [4]

\begin{tabular}{|l|l|l|l|l|l|l|l|l|c|}
\hline & -4 & -3 & -2 & -1 & 0 & 1 & 2 & 3 & 4 \\
\hline-2 & 0 & 0.0006 & -0.0001 & 0 & 0 & 0 & -0.0001 & 0.0006 & 0 \\
\hline-1 & 0.005 & $0.0429 \mathrm{j}$ & -0.1250 & $-0.2058 \mathrm{j}$ & 0.2393 & $0.2058 \mathrm{j}$ & -0.1250 & $-0.0429 \mathrm{j}$ & 0.005 \\
\hline 0 & 0 & -0.0668 & 0.0002 & 0.5644 & 1 & 0.5644 & 0.0002 & -0.0668 & 0 \\
\hline 1 & 0.005 & $-0.0429 \mathrm{j}$ & -0.1250 & $0.2058 \mathrm{j}$ & 0.2393 & $-0.2058 \mathrm{j}$ & -0.1250 & $0.0429 \mathrm{j}$ & 0.005 \\
\hline 2 & 0 & 0.0006 & -0.0001 & 0 & 0 & 0 & -0.0001 & 0.0006 & 0 \\
\hline
\end{tabular}

$h^{j i}=\left[h_{0}^{j i}, h_{1}^{j i}, \ldots \ldots \ldots, h_{i}^{j i}\right]$ characterizing the channel between the $i^{\text {th }}$ transmit and the $j^{\text {th }}$ receive antenna. The signal received by the $j^{\text {th }}$ receive antenna can be written as-

$$
r_{m}^{j}=\sum_{i=1}^{N_{t}} \sum_{p=0}^{v} h_{p}^{j i} S_{m-p}^{i}+\eta_{m}^{j}
$$

Where $\eta_{m}$ denotes the Additive White Gaussian Noise (AWGN) present at the input of the $j^{\text {th }}$ receive antenna. After downsampling by a factor $\frac{M}{2}$, the low rate signal at the output of the $K^{\text {th }}$ analysis filter of the $j^{\text {th }}$ receive antenna can be expressed as- 


$$
y_{K_{n} n}^{j}=\sum_{m=0}^{L_{p}-1} f_{K_{d} m} r_{n \frac{M}{2}-m}^{j}
$$

In case of MIMO frequency selective channels, the received signal $y_{K n}^{j}$, for $\mathrm{K} \in M_{u}$ and $\mathrm{j}=1,2, \ldots, N_{Y}$, is accompanied with other interference signals namely- ICI, ISI and inter antenna interference (IAI). The IAI refers to the mutual interference between different spatially multiplexed data streams.

\section{BASIC MIMO FBMC-OQAM APPROACHES \\ 3.1 Spatial Multiplexing}

Increasing the throughput by transmitting different data streams over different antennas.

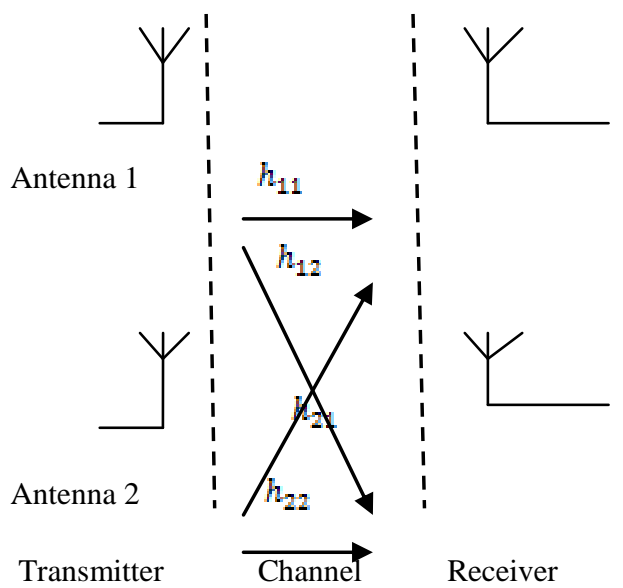

Fig 5: Example of spatial multiplexing of a MIMO $2 \times 2$ transmission[5]

\subsection{Spatial Diversity}

Improves the robustness of the transmission by transmitting the same data over different antennas with coding

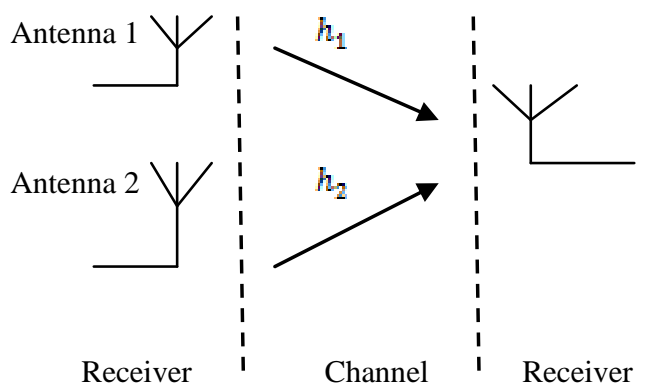

Fig 6: Example of Transmit diversity with 2 antennas[5]

\section{MIMO FBMC TECHNIQUES}

In MIMO-FBMC transmission, two situations may occur due to the possibility of FBMC to have independent or nonoverlapping subcarriers. When subcarriers-

- Do not overlap: QAM modulation is used and hence the MIMO context is similar to OFDM and same techniques can be employed.

- Overlap: Offset QAM modulation is used, therefore the OFDM technique must be adapted and specific schemes must be elaborated.

The main MIMO-FBMC techniques are given below:
A) Spatial Multiplexing

- BLAST

$>$ Diagonal BLAST (D-BLAST)

$>$ V-BLAST

B) Diversity Techniques

- $\quad$ Space Time Code(STC)

$>\quad$ Space Time Trellis Code(STTC)

$>$ Space Time Block Codes(STBC)

- $\quad$ Almouti Scheme with FBMC

- The Zero delay case

- Single delay STTC in FBMC with two antennas

\section{RECENT ADVANCEMENTS IN MIMO FBMC TECHNIQUES}

The main obstacle in the adoption of MIMO techniques by FBMC systems is the association of interference terms such as ISI, ICI and IAI in the received data which complicates its detection at the receiving end. To mitigate these interference terms, many researchers have proposed and introduced various methods that deals with - improving the decoding techniques, equalization schemes, modified and effective FBMC schemes .

\subsection{Decoding Techniques}

FBMC is based on real orthogonality, therefore its association with MIMO techniques need specific decoding schemes. A new decoding scheme is proposed based on iterative interference estimation and cancellation which does not require any channel coding or decoding block[6]. It was shown that in the case of QPSK modulation and Rayleigh channel, this decoding method of FBMC-STTC outperforms OFDM-STTC (CP taking apart) with only a slight increase in complexity.

A novel beamforming/precoding technique based on the signal to leakage plus noise ratio (SINR) is proposed in [7], inorder to mitigate the intrinsic interference as well as inter user interference in multistream MIMO-FBMC. The proposed scheme provided better performance compared to conventional MIMO-OFDM and hence proved that FBMC is a good alternative for high speed wireless communication.

A MIMO technique addressing the design of precoding and decoding technique for the FBMC modulation is proposed in [8]. This technique enabled the system to deal with the channel frequency selectivity if the channel frequency response is flat at the subcarrier level also allowing the spatial multiplexing of several streams on each subband. The simulation results confirmed that the asymmetric configurations succeed in providing similar BER results to OFDM but achieves a better spectral efficiency.

\subsection{Equalization Schemes}

In MIMO time varying systems, equalizing the channel is a challenging issue due to the distortions caused by the highly frequency selective channels. To remove these distortions, a Minimum Mean Square Error (MMSE) equalizer is derived in [3] as the classical one tap equalizer does not provide a satisfactory performance. Further to improve the performance, techniques such as successive interference cancellation (SIC), Ordered SIC(OSIC) and Two Stage OSIC (TS-OSIC) were introduced assuming spatial multiplexing is used. Simulation results confirmed the effectiveness over the classical tap equalizer. 
An adaptive T/2-spaced Decision Feedback Equalization (DFE) algorithm for MIMO-FBMC systems is presented in [9], which is both computationally efficient and numerically stable. The algorithm is based on the well kown equivalence of the V-BLAST idea with the generalised DFE [10]. It updates both the filter taps and the BLAST ordering in an efficient RLS based manner. The simulation results demonstrated its effectiveness in scenarios of varying fading rate and frequency selectivity.

\subsection{Modified and Effective FBMC Schemes}

(a) Although FMT based FBMC system is less bandwidth efficient compared to other FBMC systems, is still attractive due to its compatibility with MIMO channels. So, a novel filter bank design is developed in [11] that minimizes the bandwidth loss. This isotropic filter is designed using the design procedure proposed by Haas and Belfiore [12],[13] and further modifications were made. The resulting filter were more robust to doubly spread effects of channel and was able to achieve densities of higher than $1 / \mathrm{TF}=1 / 2$. The work in [11] was further extended in [14] where further improvement was achieved by organizing the data symbols in a hexagonal lattice structure, as compared to the more popular rectangular structure.

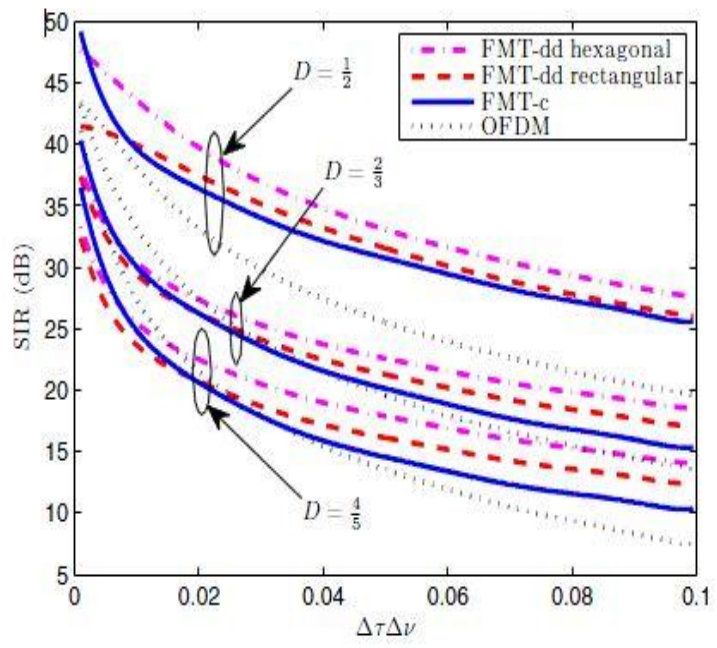

Fig 7: The ambiguity function of the prototype filter designed for the density $1 / \mathrm{TF}=1 / 2[14]$.

The results comparing the performance of conventional FMT (FMT-c) and the later modified FMT (FMT-dd) is presented in Fig.7 and the following results were observed-

- In high mobility environments, FMT-dd designs significantly outperform OFDM and FMT-c.

- The hexagonal lattice based isotropic filter designs perform around $2 \mathrm{~dB}$ better than their rectangular lattice counterparts.

- $\quad$ As density D decreases, the performance of FMT-c approaches that of the FMT-dd with rectangular lattice.

(b) Inorder to get rid of the intrinsic interference which is an issue while combining FBMC with some MIMO techniques such as Spatial Multiplexing- Maximum Likelihood Detection (SM-MLD) and Almouti coding, a new FBMC scheme called FFT-FBMC have been proposed in [15]. This scheme is computationally more complex than FBMC. The transmission strategy used in this scheme is given below in Table.2.
Table 2: Data transmisssion strategy within a data block[15]

Block of $\mathrm{N}$ symbols

\begin{tabular}{|c|c|}
\hline \multicolumn{2}{|c|}{ Double rate data } \\
\hline No data & Double rate data \\
\hline Double rate data & No data \\
\hline No data & Double rate data \\
\hline $\mathbf{I}$ & $\mathbf{1}$ \\
\hline
\end{tabular}

In this transmission strategy, no data is transmitted on the faded positions and modulation rate is doubled on the remaining positions of each subcarrier. Therefore the resulting system became formulated as OFDM, and all MIMO techniques can be applied in a straightforward manner.

(c) Similarly as a remedy to the intrinsic interference in MIMO FBMC/OQAM systems, widely linear processing in FBMC/OQAM systems have been brought into attention in previous works such as [16]. It is also shown in [17] that mitigating the intrinsic interference enables the application of maximum likelihood (ML)detection in MIMO transmissions with FBMC/OQAM. Further in [18], a two step receiver is developed for a point to point MIMO FBMC/OQAM systems where two schemes of estimating the intrinsic interference are presented and analysed via simulations. First scheme where an estimate of the intrinsic interference is obtained by directly taking the imaginary part of the output of the linear MMSE receiver and second scheme uses the detected adjacent multicarrier symbols. The two Step receiver with the second scheme achieved a very promising performance. However, some delay is incurred since the knowledge of the detected future symbols is required.

(d) CMT based FBMC system was identified and introduced in[19] as the best choice for application in massive MIMO because of its blind equalization capability. The numerical results presented in [19] satisfied the claim that apart from offering the same processing gain as OFDM, FBMC-CMT also offers the advantages of-

- More flexible carrier aggregation

- Higher bandwidth efficiency

- Larger subcarrier spacing

- Less sensitivity to CFO

- Lower PAPR

\section{CONCLUSION}

The paper presented the study of various design schemes/methods of FBMC systems that enhances its flexibility while adopting MIMO technology. The ISI and ICI signals which are the major bottleneck in MIMO-FBMC systems are mitigated using efficient decoding and equalization schemes. Further, modifications in FBMC systems such as FMT and CMT were shown making them more compatible with MIMO techniques. The study show that MIMO-FBMC could stand as a good alternative to MIMOOFDM. 


\section{ACKNOWLEDGEMENT}

The authors gratefully acknoweledge and would like to thank Dr. Sonika Singh for the valuable comments and suggestions that helped in improving this paper.

\section{REFERENCES}

[1] International Telecommunication Union radio communication "Assessment of the global mobile broadband deployments and forecasts for mobile telecommunications," ITU-R M.2243,2011.

[2] FPT-ICT Project PHYDYAS- Physical layer for dynamic spectrum access and cognitive radios. http://www.ictphydyas.org.

[3] Aissa Ikhlef and Jerome Louveaux "Per subchannel equalization for MIMO FBMC/OQAM systems," Proceedings of the IEEE,Communications, Computers and Signal Processing, pp:559-564,2009.

[4] M. Bellanger, "Specifications and design of a prototype filter for filterbank based multicarrier transmission," vol.4,2001.

[5] FPT-ICT Project PHYDYAS- Physical layer: a primer. http://www.ict-phydyas.org.

[6] C. Lele, D. Le Ruyet, R. Zakaria,"On the decoding of single delay STTC using filter bank based multicarrier modulation," In proceding of:Wireless Communication Systems,2009.ISWCS 2009.

[7] Upul Jayasinghe, Nandana Rajatheva, and Matti Latvaaho,"Leakage based Mulit User Beamforming Scheme to mitigate interference in MIMO-FBMC," Smart Antennas(WSA ),IEEE Conference,pp.1-5,2013.

[8] Marius Caus and Ana I. Perez-Neira," Multistream transmission in MIMO-FBMC systems,"Speech and Signal Processing(ICASSP), IEEE Conference ,pp.50415045,2013

[9] Eleftherios Kofidis and Athanasios A.Rontogiannis, "Adaptive BLAST decision feedback equalizer for MIMO-FBMC/OQAM systems," PIMR,IEEE $21^{\text {st }}$ Symposium,pp.841-846,2010.
[10] G. Gini and J.M. Cioffi, "On the relation between VBLAST and the GDFE," IEEE Communicatios Letters;vol.5,no.9, pp.364-366, Sept.2001.

[11] Pooyan Amini, Chung Him Yuen, Rong-Rong Chen, and Behrouz Farhang-Boroujeny, "Isotropic filter design for MIMO filter bank multicarrier communications,"SAM,IEEE.pp.89-92,2010.

[12] M. Alard," Construction of a multicarrier signal," Patent WO 96/35278, 1996

[13] R. Hars and J. C. Belfiore," A time frequency well localized pulse for multiple carrier transmission," Wireless personal communications, vol.5,pp.1-18,1997.

[14] Pooyan Amini, and Behrouz Farhang-Boroujeny," Design and performance evaluation of Filtered Multitone(FMT) in doubly dispersive channels,'ICC,IEEE International Conference,pp.15,2011 .

[15] R.Zakaria, and D. Le Ruyet, "A novel scheme for spatial multiplexing with maximum likelihood detection,'ISWCS, pp.461-465.2010.

[16] M. Caus and A. I. Perez- Neira, "Multistream transmissions in MIMO-FBMC systems," in Processing IEEE International Conference on Acoustics, Speed and signal processing (ICASSP), May 2013.

[17] R. Zakaria, D. Le Ruyet, and M. Bellenger, "Maximum Likelihood detection in spatial multiplexing with FBMC," in Processing 2010 European Wireless, June 2010.

[18] Yao Cheng and Martin Haardt, "Widely Linear Processing in MIMO FBMC/OQAM ystems," ISWCS,IEEE Proceedings,pp.1-5,2013.

[19] Arman Farhang, Nicola Marchetti, Linda E. Doyle and Behrouz Farhang-Boroujeny, "Filter bank multicarrier for massive MIMO," arXiv:1402.5881 [cs.IT]pp.17,2014 . 